\title{
Robotically assisted laparoscopic repair of anterior vaginal wall and uterine prolapse by lateral suspension with mesh: initial experience and video
}

\author{
Patrick Dällenbach • Nikolaus Veit
}

Received: 11 November 2013 / Accepted: 1 February 2014 / Published online: 17 April 2014

(C) The International Urogynecological Association 2014

\begin{abstract}
Introduction and hypothesis Sacral colpopexy/hysteropexy is a well-established approach to vaginal apex support and was the first technique used to treat pelvic organ prolapse (POP) with robotic assistance. However, dissection at the level of the promontory may be difficult, especially in obese patients, and associated with rare but potential serious morbidity such as life-threatening vascular injury. In an attempt to avoid this risk, we describe a new robotic approach for POP repair with lateral suspension. Methods From March 2012 through June 2013, ten patients with symptomatic anterior vaginal wall and uterine prolapse were operated by a single surgeon. The video presents the different steps of robotically assisted laparoscopic repair of POP by lateral suspension with mesh and uterine conservation using da Vinci $\mathrm{S}$ or Si system.

Results POP repair was successfully completed in all ten patients without any perioperative or postoperative complication. Conclusion Robotically assisted laparoscopic repair of POP by lateral suspension with mesh is a novel and feasible technique with promising short-term results. It may have several theoretical advantages over sacral colpopexy/hysteropexy and may represent an alternative in cases of difficult dissection of the promontory.
\end{abstract}

Electronic supplementary material The online version of this article (doi:10.1007/s00192-014-2349-2) contains supplementary material, which is available to authorized users. This video is also available to watch on http://videos.springer.com/. Please search for the video by the article title.

P. Dällenbach · N. Veit

Department of Gynecology and Obstetrics, Division of Gynecology, Geneva University Hospitals, Geneva, Switzerland

P. Dällenbach $(\triangle)$

Department of Gynecology and Obstetrics, Division of Gynecology, Urogynecology Unit, Geneva University Hospitals, 30 boulevard de la Cluse, 1211 Genève 14, Switzerland

e-mail: Patrick.Dallenbach@hcuge.ch
Keywords Pelvic organ prolapse · Sacrocolpopexy · Hysteropexy · Robotic surgery $\cdot$ da Vinci system . Uterine prolapse

\section{Aim of the video}

Sacral colpopexy/hysteropexy is the method of choice for the treatment of apical vaginal and uterine prolapse. It was the first technique described to treat pelvic organ prolapse (POP) with robotic assistance. It offers similar results as abdominal sacrocolpopexy [1]. Access to robotic technology may make conversion from open to laparoscopic surgery more feasible for most pelvic floor surgeons. However, sacrocolpopexy is associated with rare but serious morbidity, with case reports of life-threatening vascular injuries at the level of the promontory, vertebral osteomyelitis, and nerve injuries [2]. In 1967, Kapandji described an alternative technique with lateral suspension of apical prolapse, avoiding dissection of the promontory, thus reducing the risks associated with sacrocolpopexy [3]. The technique consisted of fixation of the anterior vagina and isthmus of uterus with a mesh to the anterior superior iliac bone. In 1994, Cornier and Madelenat described a new development based on Kapandji's technique by laparoscopy [4]. The technique was progressively modified by Dubuisson et al., with a higher transparietal tension-free lateral suspension, $5 \mathrm{~cm}$ above the anterior superior iliac spine [5]. Published case series show similar results to those of sacrocolpopexy in terms of safety and efficiency, with low complication rates and no serious adverse events. We further developed this technique with the da Vinci system which allowed us to avoid the transparietal passage of the mesh of the laparoscopic technique, therefore reducing the number of scars and avoiding potential damage to the ilioinguinal and iliohypogastric nerves. The goal of our video and our manuscript is to highlight the technique and give preliminary results. 


\section{Method}

From March 2012 to July 2013, we performed robotically assisted laparoscopic repair of POP by lateral suspension with mesh in ten patients with symptomatic anterior and apical POP stage II or more. All women had a standardized preoperative prolapse assessment, using the Pelvic Organ Prolapse Quantification system (POP-Q) classification and preoperative urodynamics. We informed all patients of the novelty of the procedure. All ten patients gave their informed consent. The surgical technique was the same in all patients and performed by one surgeon (P.D.).

We used either da Vinci S or Si system (Intuitive Surgical $\AA$ ) to perform the procedure as they were the two models available in our institution during that period. All patients had preoperative prophylactic antibiotics (Mefoxitin $2 \mathrm{~g}$ intravenously) at induction of anesthesia and were placed in the dorsal lithotomy position. We used one intrauterine curette inserted together with two tenacula fixed to the cervix to manipulate and expose the uterus by an assistant sitting between the legs of the patient. We performed insufflation with a Veress Needle. A 12-mm trocar (Ethicon ${ }^{\circledR}$ D12 XT) $150 \mathrm{~mm}$ long was introduced intraumbilically or supraumbilically on the midline depending on the anatomy of the patient. After introducing the da Vinci $0^{\circ}$ optic, we placed the two metallic da Vinci 8-mm trocars in each iliac fossa, laterally about $5 \mathrm{~cm}$ above and $2 \mathrm{~cm}$ medial to the anterior superior iliac spine. We introduced another $10-\mathrm{mm}$ trocar paraumbilically for the field assistant between the port of the camera and the 8-mm port of the right iliac fossa. After diagnostic laparoscopy, the da Vinci robot was docked between the legs of the patient. The robotic procedure started with dissection of the uterovesical pouch with da Vinci bipolar Maryland forceps and monopolar curved scissors. Dissection was helped by mobilization of the uterus and exposure of the anterior vaginal wall with a metallic malleable parallel retractor introduced into the vagina by the assistant. A swab was introduced intra-abdominally to help hemostasis and vesicovaginal dissection and removed after the end of the dissection. Dissection was stopped just above the level of the bladder neck. A polypropylene mesh (TiLOOP ${ }^{\circledR}$ "Prof Dubuisson" ${ }^{\circledR}$ 9X $41.5 \mathrm{~cm}, 65 \mathrm{~g} / \mathrm{m}^{2}$ ) with two lateral arms of $3 \mathrm{~cm}$ width with an anterior vaginal part of $6 \mathrm{~cm}$ length and $5 \mathrm{~cm}$ width was introduced through the $10-\mathrm{mm}$ paraumbilical port and fixed to the vagina by four to six sutures of $2-0$ polyglactin 910 (Vicryl ${ }^{\circledR}$ suture 2-0, V3170H, JB needle by Ethicon). The mesh was also fixed to the anterior cervix and to the isthmus of uterus. We only used absorbable sutures on the vagina. We believe that it is the inflammatory reaction mediated by the mesh which is responsible for the long-term support and that nonabsorbable sutures are unnecessary. Once the mesh was sutured, we made a peritoneal incision on the lateral abdominal wall about $3 \mathrm{~cm}$ above the anterior and superior iliac spine using monopolar scissors, just at the end of the lateral ports. One of the lateral arms of the robot was undocked by the operating field assistant allowing him to introduce a laparoscopic forceps through the 8-mm port. This instrument was introduced through the peritoneal incision and oriented retroperitoneally under the round ligament to grasp the lateral arm of the mesh. The arm was pulled out slowly by the same way until satisfactory tension was obtained. Excess mesh material was cut and removed. The same procedure was performed on the opposite side. Then, the peritoneum of the vesicouterine fold was closed over the mesh with uninterrupted suture of polyglactin 910 (Vicryl ${ }^{\circledR}$ suture 0 CT-2 needle, V330). During the closure, we performed a plication of the round ligaments and included the mesh in the suture, thus increasing the binding of the mesh to the uterus and to the round ligaments. After checking the result of the procedure and avoiding excessive tension, the arms of the mesh were fixed to the peritoneum of the abdominal wall by the field assistant with absorbable tacks (AbsorbaTack ${ }^{\circledR}$ fixation device by Covidien). After cleaning the abdominal cavity and checking both ureters, the procedure was concluded. For both 12-mm trocar incisions, we closed the fascia before closing the skin. The video illustrates the technique and combines the sequences of two women who suffered from anterior and apical POP stage III and no posterior prolapse. We performed descriptive statistics alone. Data were managed and analyzed with SPSS18.0 statistical software (SPSS Inc., Chicago, IL, USA).

\section{Results}

POP repair was successfully completed in all ten patients. There was no intra- or postoperative complication. Mean age was 57.1 (SD 6.5) years (range 41.0-63.0 years) and mean body mass index (BMI) 25.8 (SD 3.6) (range 22.1-32.9). Seven patients had previous abdominal surgery $(70 \%)$ but none had previous hysterectomy. One patient had previous POP surgery (posterior colpoperineorrhaphy) and previous operation for urinary stress incontinence (laparoscopic Burch colposuspension). The median total duration time of the operation was 206.5 min (interquartile range 190.3-251.0). Mean blood loss was 8 (SD 16.2) $\mathrm{ml}$ (range 0-50 ml). The postoperative course was uneventful. All patients returned home between the third and fifth postoperative days. There was no recurrence (defined as POP stage I or more), erosion, de novo urgency, or urinary incontinence at short-term follow-up [mean 52.8 (SD 20.2) days].

\section{Conclusion}

Robotically assisted laparoscopic repair of POP by lateral suspension with mesh is a novel and feasible technique with promising short-term results for the treatment of anterior vaginal wall and uterine prolapse. By avoiding promontory dissection, it may have several theoretical advantages over 
sacral colpopexy/hysteropexy, including reduction of the risk of vascular and nerve injuries as well as vertebral osteomyelitis. It may represent a useful alternative in cases of pelvic kidney, bony abnormalities, and difficult promontory dissection such as fatty presacral space with difficulties reaching the longitudinal ligament. When compared to the initial laparoscopic technique using transparietal pulling out of the mesh arms, robotic assistance, by avoiding this step, may help avoid potential abdominal wall nerve damage. However, in both methods the technical aspects of the lateral suspension are similar, and we believe long-term results to be comparable with a $5 \%$ rate of reoperation for recurrence in a laparoscopic study of 218 patients [5]. Further studies with longer followup are needed to confirm these preliminary results.

\section{Consent}

Written informed consent was obtained from the patients for publication of this video article and any accompanying images.
Conflicts of interest None.

\section{References}

1. Geller EJ, Parnell BA, Dunivan GC (2012) Robotic vs abdominal sacrocolpopexy: 44-month pelvic floor outcomes. Urology 79:532536

2. Nygaard IE, McCreery R, Brubaker L, Connolly A, Cundiff G, Weber AM, Zyczynski H et al (2004) Abdominal sacrocolpopexy: a comprehensive review. Obstet Gynecol 104:805-823

3. Kapandji M (1967) Treatment of urogenital prolapse by colpoisthmo-cystopexy with transverse strip and crossed, multiple layer, ligamento-peritoneal douglasorrhaphy. Ann Chir 21:321328

4. Cornier E, Madelenat P (1994) The M. Kapandji hysteropexy: a laparoscopic technic and preliminary results. J Gynecol Obstet Biol Reprod (Paris) 23:378-385

5. Dubuisson JB, Eperon I, Jacob S, Dubuisson J, Wenger JM, Dallenbach P, Kaelin-Gambirasio I (2011) Laparoscopic repair of pelvic organ prolapse by lateral suspension with mesh: a continuous series of 218 patients. Gynecol Obstet Fertil 39: $127-131$ 